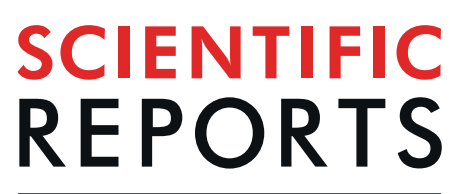

natureresearch

\title{
OPEN Compton profile of few-layer graphene investigated by electron energy-loss spectroscopy
}

\author{
Zhenbao Feng ${ }^{1 *}$, Xiaoyan Zhang ${ }^{1}$, Yoshiharu Sakurai ${ }^{2}$, Zongliang Wang ${ }^{1}, \mathrm{Hefu} \mathrm{Li}^{1}$ \& \\ Haiquan $\mathrm{Hu}^{1}$
}

In this paper, acquisition of the valence Compton profile of few-layer graphene using electron energyloss spectroscopy at large scattering angle is reported. The experimental Compton profile is compared with the corresponding theoretical profile, calculated using the full-potential linearized augmented plane wave method based on the local-density approximation. Good agreement exists between the theoretical calculation and experiment. The graphene profile indicates a substantially greater delocalization of the ground state charge density compared to that of graphite.

Electron energy-loss spectroscopy (EELS) is a powerful tool to study the electronic structure properties of solids ${ }^{1}$. The quantity measured in an EELS experiment is the double differential scattering cross-section ${ }^{2}$

$$
\frac{d^{2} \sigma}{d \Omega d E}=\frac{4 \gamma^{2} R}{E q^{2}} \frac{k}{k_{0}} \frac{d f}{d E}(q, E)
$$

where $\gamma$ is the relativistic factor; $R$ is the Rydberg energy; $k_{0}$ and $k$ are wavevectors of the fast electron before and after scattering, respectively; $\mathrm{d} f / \mathrm{d} E$ is the generalized oscillator strength (GOS) that is a function of the momentum transfer $\mathbf{q}$ and the energy loss $E$. The physical information of the GOS depends on the $\mathbf{q}$ and $E$ regimes ${ }^{2-5}$. For small $q$, the GOS contains information about the unoccupied density of states: On the one hand, when $q \cdot r \ll 1$, where $\mathbf{r}$ represents the electron orbital radius, the dipole-allowed transitions dominate the energy-loss spectrum. On the other hand, when $q \cdot r \geq 1$, monopolar and higher-order transitions become important. The EELS recording in this momentum transfer regime is known as the electron energy-loss near-edge structure (ELNES). For large momentum transfer relative to the inverse electronic orbital size, and for large energy loss (transfer) relative to the binding energy of an electron; the impulse approximation is valid ${ }^{6,7}$. The GOS provides information about the ground-state electron momentum density, known as electron Compton scattering from solids $(\mathrm{ECOSS})^{8}$. The EELS is acquired by tilting the incident beam through a few degrees $\left(\sim 5^{\circ}\right)$ for ECOSS measurement so that only large-angle-scattered electrons are collected ${ }^{9,10}$. The ECOSS technique has proven to be an important alternative for experimentally investigating the electron momentum density distribution of nanomaterials ${ }^{11,12}$.

The past few years have seen a prodigious amount of research on nanocarbon materials, and on graphene in particular ${ }^{13-15}$. In general, understanding the many fascinating properties of graphene requires a detailed knowledge of its electronic structure. Although sufficient work is available regarding the structural, electronic and optical properties of graphene, the experiment and theoretical electron momentum density of graphene still remains unexplored $^{16-21}$.

Compton scattering is a well-established and unique tool to study the electronic structure of materials via their electron momentum density ${ }^{22-26}$. The shape of the Compton profile is sensitive to the state of the valence electrons of the atoms which combine to form the condensed phase of the material ${ }^{7}$. It offers a straight way to test the band structure calculations for different materials ${ }^{23,25}$. Since most of the theoretical studies of the electronic structure of graphene have been carried out using the density functional theory (DFT) and the local density approximation (LDA), it is important to test the applicability of these approaches. In this work, the Compton scattering experiments on graphene are reported using the ECOSS technique. The valence Compton profile of graphene is obtained by subtracting the theoretical core-electron Compton scattering from the experimental profile. In

${ }^{1}$ School of Physics Science and Information Technology, Shandong Key Laboratory of Optical Communication Science and Technology, Liaocheng University, Liaocheng, 252059, China. ${ }^{2} J a p a n$ Synchrotron Radiation Research Institute, SPring-8, 1-1-1 Kouto, Sayo, Hyogo, 679-5198, Japan. *email: fengzhenbao@lcu.edu.cn 


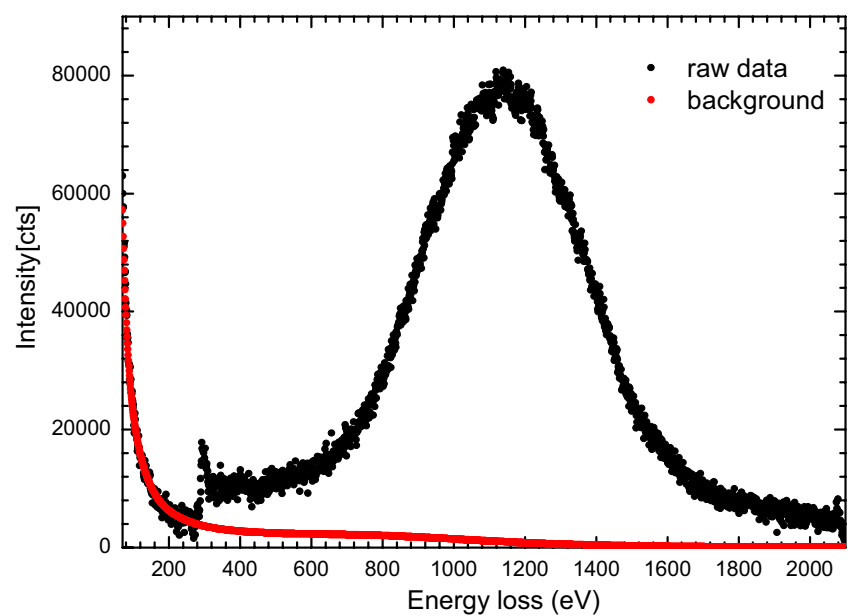

Figure 1. Electron energy-loss spectrum (EELS) of few-layer graphene at $\sim 73 \mathrm{mrad}$ scattering angle with a $30 \mathrm{~s}$ exposure time (black dots), along with the simulated elastic-inelastic scattering background (red dots).

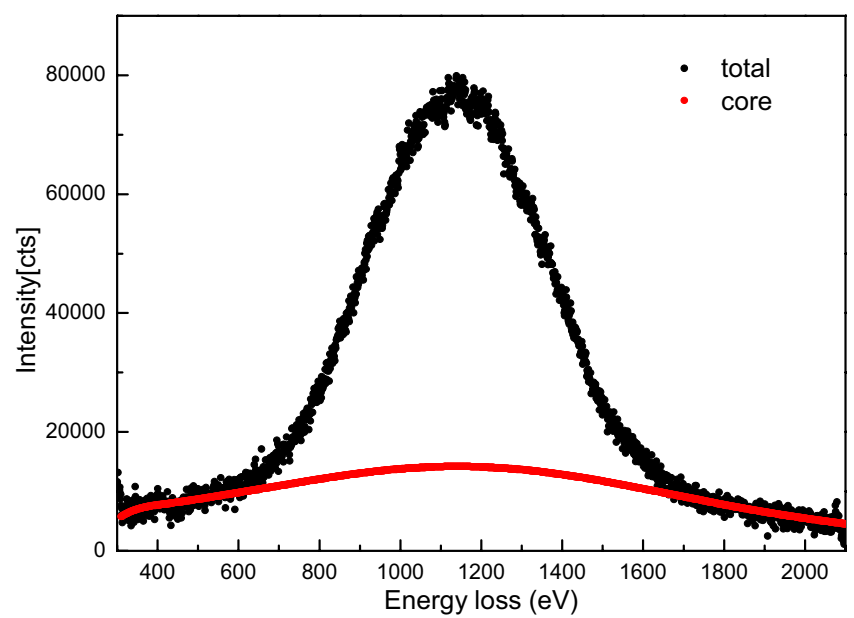

Figure 2. Measured EELS after background subtraction (black dots) and the contribution from the core Compton scattering as determined by Hartree-Slater method (red dots).

addition, we calculated the valence Compton profile of graphene using a full-potential linearized augmented plane wave (FLAPW) within the framework of the density functional theory ${ }^{27}$. The experimental results are in excellent agreement with the theoretical Compton profile of graphene. Further, the graphene Compton profile is compared with that of graphite both experimentally and theoretically, where a reasonable agreement between experiment and theory was achieved.

\section{Results and Discussion}

The raw electron Compton scattering spectrum of graphene by recording EELS at the scattering angle of $\sim 73$ mrad is shown in Fig. 1. It is worth pointing out that the directions of momentum transfers predominantly lay in the basal plan at this large scattering angle. We thus predominantly probed the basal plan Compton profile of graphene in this experiment. The Compton scattering spectrum extends over hundreds of electron volts, so the typical power-law background subtraction method does not work. In this work, the background of the ECOSS spectrum was subtracted by parameterized simulations of combined elastic and inelastic scattering events ${ }^{28}$. A characterization angle of $15 \mathrm{mrad}$ and the ratio of quasi-elastic to inelastic scattering cross-sections of 0.8 were chosen for the background simulation. The core Compton scattering spectrum as a function of energy loss $(\mathrm{eV})$ was determined using the Hartree-Slater method at a momentum transfer of 9.25 a.u., as shown in Fig. 2. We note that the theoretical core Compton scattering spectrum is in good agreement with the experimental profile at both the leading and trailing edges. This shows the rationality of our background subtraction ${ }^{29}$.

Figure 3 shows the measured and calculated valence Compton profiles $\left(J_{\mathrm{v}}\left(p_{q}\right)\right)$ of graphene as a function of momentum $\left(p_{q}\right)$. The theoretical profile was convolved with the experimental resolution function. Details of our experimental data processing procedure have been previously described ${ }^{30}$. The Compton profile of graphene was calculated as a longer lattice constant along the $c$-axis $(20 \AA)$, while graphite was $6.77 \AA$. In order to compare 


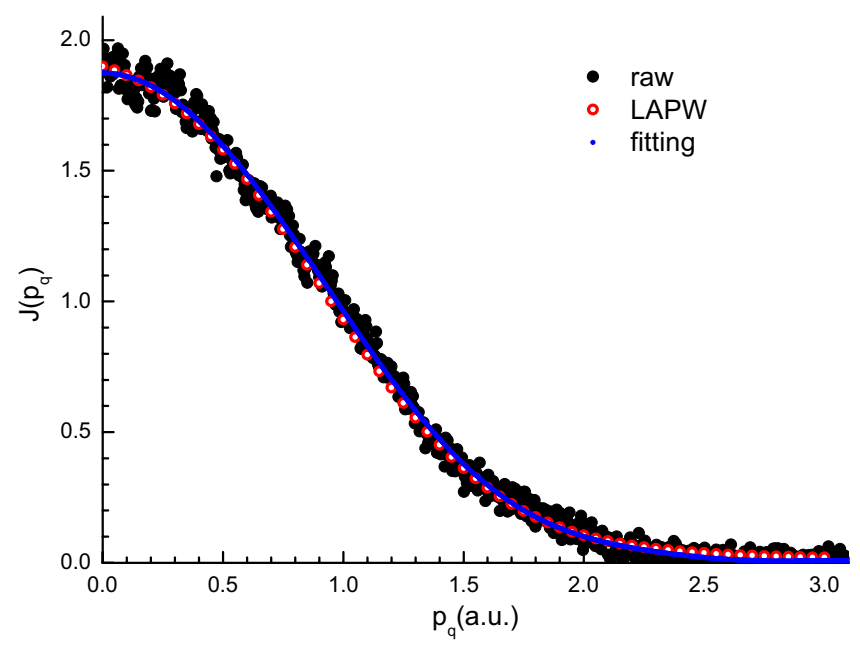

Figure 3. Experimental valence Compton profile of few-layer graphene obtained by the TEM (black dots), the polynomial fitting of the experimental data (blue line), and the FLAPW calculated results (red line).

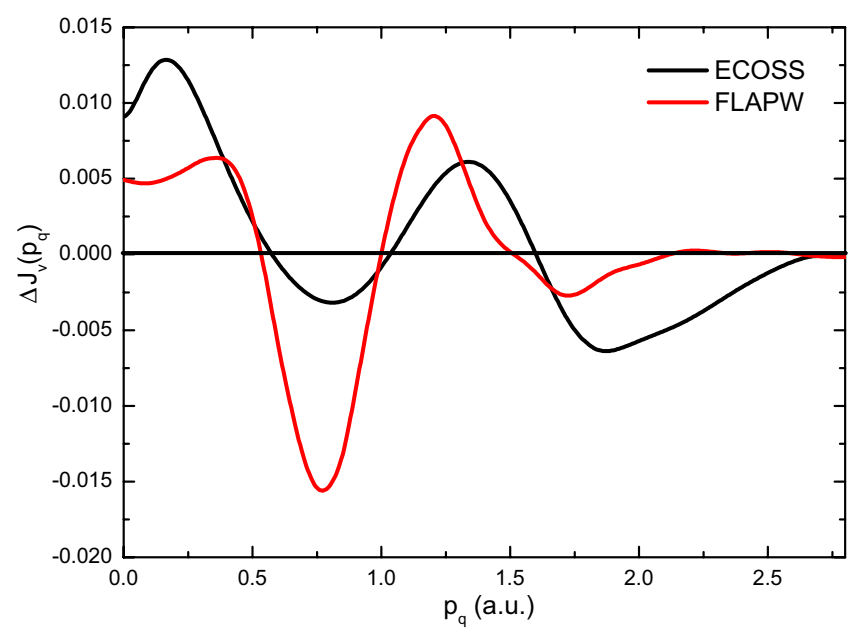

Figure 4. Comparison of the Compton profile difference for graphene and graphite, $\Delta J_{\mathrm{v}}\left(p_{q}\right)=J_{\mathrm{v}}^{\text {graphene }}\left(p_{q}\right)-J_{\mathrm{v}}^{\text {graphite }}\left(p_{q}\right)$, obtained by ECOSS experiment and FLAPW calculations.

experimental results with our calculations, the theoretical in-plane profile was obtained by averaging the Compton profiles calculated for [10 $\overline{1} 0]$ and $[11 \overline{2} 0]$ directions. The excellent agreement between the calculated and measured profiles is evident in the Fig. 3. Compton profile differences are useful for comparing experiment and theor $y^{7,31}$. The experimental Compton profile of graphite was obtained under the same experimental conditions as that of graphene by ECOSS technique ${ }^{30,32}$. Figure 4 plots the differences $\left(\Delta J_{\mathrm{v}}\left(p_{q}\right)\right)$ between the graphene $\left(J_{\mathrm{v}}^{\text {grephene }}\left(p_{q}\right)\right)$ and graphite $\left(J_{\mathrm{v}}^{\text {graphite }}\left(p_{q}\right)\right)$ profiles for both the FLAPW theoretical calculations and the ECOSS measurements. Good agreement is found in the Fig. 4 between the FLAPW calculated differences and the ECOSS measured differences, where the experimental and calculated $\Delta J_{\mathrm{v}}\left(p_{q}\right)$ exhibit similar trends. The dip near $p_{q}=0.75$ a.u. may arise from $\pi$-bonding anisotropy in graphite ${ }^{33}$. The observed narrowness of the graphene Compton profile with respect to that of graphite can be interpreted as a larger delocalization of valence electrons in the graphene than in graphite. In graphite, the electrons form $\pi$ bonds between two layers via the weak van der Walls forces. In graphene, however, these $\pi$ bonds are incomplete and the electrons forming such bonds behave almost as delocalized electrons, associated with the curving graphene layer by means of dangling bonds.

\section{Conclusions}

To summarize, the valence Compton profile of few-layer graphene was obtained by measuring the EELS in a TEM and comparing it with the FLAPW calculation based on the local-density approximation band theory framework. The experimental results are in good agreement with the theoretical calculations. The differences between the Compton profiles of graphene and graphite obtained both experimentally and via FLAPW calculations showed good agreement. Both the experimental and theoretical results indicate that the ground-state electronic density is more delocalization in graphene than in graphite. 


\section{Experimental}

The experiments were performed with a transmission electron microscope (TEM; FEI TECNAI F20, Thermo Fisher Scientific) equipped with an imaging filter (Gatan Tridiem), where the TEM was operated at an accelerating voltage of $120 \mathrm{kV}$. Commercially-available 300-mesh copper TEM grids with amorphous holey carbon film supporting transferred graphene (Graphene Supermarket, https://graphene-supermarket.com) were used, where the graphene was produced by chemical vapor deposition and consisted of 1-6 layers. The EELS technique was used to measure the electron Compton scattering of the few-layer graphene film suspended over the TEM holey carbon film holes, where the TEM was in the diffraction mode with a parallel incident beam. Though the operating energy of the incident beam was well above the knock-on threshold of graphene, the dose was spread over a sufficiently large area so that the electron beam damage to the samples was not expected to be significant over the short acquisition times $(30 \mathrm{~s})$ used herein ${ }^{34}$. The camera length was $300 \mathrm{~mm}$ and the spectrometer entrance was set to $2.5 \mathrm{~mm}$. Further, the collection semi-angle was $2.44 \mathrm{mrad}$ and the corresponding momentum transfer uncertainty was 0.16 a.u. The energy resolution was determined from the full width at half maximum of the zero-loss peak at $3 \mathrm{eV}$, which when converted to a momentum resolution was found to be $0.012 \mathrm{a}$.u. The total momentum resolution was estimated to be on the order of 0.1 a.u. ${ }^{10}$. To reduce the statistical error, more than five spectra were acquired from the sample, whereupon additional measurements were obtained to determine morphological variations on the sample to ensure that serious radiation damage had not occurred.

Received: 20 August 2019; Accepted: 28 October 2019;

Published online: 21 November 2019

\section{References}

1. Keast, V. J., Scott, A. J., Brydson, R., Williams, D. B. \& Bruley, J. Electron energy-loss near-edge structure — a tool for the investigation of electronic structure on the nanometre scale. J. Microsc. 203, 135-175 (2001).

2. Egerton, R. F. Electron energy-loss spectroscopy in electron microscope. Third edn, (Springer, New York 2011).

3. Nelhiebel, M. et al. Theory of orientation-sensitive near-edge fine-structure core-level spectroscopy. Phys. Rev. B 59, 12807-12814 (1999).

4. Mattern, B. A., Seidler, G. T., Kas, J. J., Pacold, J. I. \& Rehr, J. J. Real-space Green's function calculations of Compton profiles. Phys. Rev. B 85, 115135 (2012).

5. Sternemann, C. et al. Momentum-transfer dependence of X-ray Raman scattering at the Be K-edge. Phys. Rev. B 68, 035111 (2003).

6. Williams, B. G. \& Bourdillon, A. J. Localized Compton scattering using energy-loss spectroscopy. J Phys C Solid State 15, 6881-6890 (1982).

7. Williams, B. G. Compton Scattering. (McGraw-Hill, New York 1977).

8. Williams, B. G., Sparrow, T. G. \& Egerton, R. F. Electron Compton scattering from solids. Proc. R. Soc. Lond. A 393, 409-422 (1984).

9. Williams, B. G., Parkinson, G. M., Eckhardt, C. J., Thomas, J. M. \& Sparrow, T. A new approach to the measurement of the momentum densities in solids using an electron microscope. Chem. Phys. Lett. 78, 434-438 (1981).

10. Jonas, P. \& Schattschneider, P. The experimental conditions for Compton scattering in the electron microscope. J. Phys.: Condens. Matter 5, 7173-7188 (1993).

11. Feng, Z. B., Yang, B., Lin, Y. M. \& Su, D. S. Investigation of the electron momentum density distribution of nanodiamonds by electron energy-loss spectroscopy. J. Chem. Phys. 143, 211102 (2015).

12. Feng, Z. B., Lin, Y. M., Tian, C. W., Hu, H. Q. \& Su, D. S. Combined study of the ground and excited states in the transformation of nanodiamonds into carbon onions by electron energy-loss spectroscopy. Sci Rep-Uk 9, 3784 (2019).

13. Georgakilas, V., Perman, J. A., Tucek, J. \& Zboril, R. Broad family of carbon nanoallotropes: classification, chemistry, and applications of fullerenes, carbon dots, nanotubes, graphene, nanodiamonds, and combined superstructures. Chem. Rev. 115, 4744-4822 (2015).

14. Lin, Y. M. et al. Insights into the surface chemistry and electronic properties of $\mathrm{sp}^{2}$ and $\mathrm{sp}^{3}$-hybridized nanocarbon materials for catalysis. Chem. Commun. 53, 4834-4837 (2017).

15. Ferrari, A. C. et al. Raman spectrum of graphene and graphene layers. Phys. Rev. Lett. 97, 187401 (2006).

16. Hua, W. J., Gao, B., Li, S. H., Agren, H. \& Luo, Y. X-ray absorption spectra of graphene from first-principles simulations. Phys. Rev. $B$ 82, 155433 (2010).

17. Suenaga, K. \& Koshino, M. Atom-by-atom spectroscopy at graphene edge. Nature 468, 1088-1090 (2010).

18. Schultz, B. J. et al. Imaging local electronic corrugations and doped regions in graphene. Nat Commun 2,372 (2011).

19. Nejati, H. \& Dadsetani, M. Electron energy loss spectrum of graphane from first-principles calculations. Micron 67, 30-36 (2014).

20. Liou, S. C. et al. $\pi$-plasmon dispersion in free-standing graphene by momentum-resolved electron energy-loss spectroscopy. Phys. Rev. B 91, 045418 (2015).

21. Mohn, M. J., Hambach, R., Wachsmuth, P., Giorgetti, C. \& Kaiser, U. Dielectric properties of graphene/MoS2 heterostructures from ab initio calculations and electron energy-loss experiments. Phys. Rev. B 97, 235410 (2018).

22. Cooper, M. J. Compton scattering and electron momentum determination. Rep. Prog. Phys. 48, 415-481 (1985).

23. Cooper, M. J., Mijnarends, P. E., Shiotani, N., Sakai, N. \& Bansil, A. X-Ray Compton scattering. (Oxford University Press, 2004).

24. Manninen, S. Compton scattering: present status and future. J. Phys. Chem. Solids 61, 335-340 (2000).

25. Sharma, G. et al. Electron momentum density in multiwall carbon nanotubes. Physica E-Low-Dimensional Systems \& Nanostructures 43, 1084-1086 (2011).

26. Feng, Z. B. et al. Investigation of electron momentum density in carbon nanotubes using transmission electron microscopy. Microsc. Microanal. 25, 1155-1159 (2019).

27. The code BANDS01 was supplied by Mizuho Information \& Research Institute, Inc. (2004).

28. Su, D. S., Jonas, P. \& Schattschneider, P. The multiple-scattering problem in electron Compton scattering on solids. Philos. Mag. B 66, 405-418 (1992).

29. Mijnarends, P. E. \& Bansil, A. Encyclopedia of condensed matter physics "Scattering techniques, Compton". 1st edn, (Elsevier 2005).

30. Feng, Z. B. et al. Combined study of the ground and unoccupied electronic states of graphite by electron energy-loss spectroscopy. J. Appl. Phys. 114, 183716 (2013).

31. Matsuda, K. et al. Electron momentum density in liquid silicon. Phys. Rev. B 88, 115125 (2013).

32. Feng, Z. B., Sakurai, Y., Liu, J. F., Su, D. S. \& Schattschneider, P. Anisotropy of electron Compton profiles of graphite investigated by electron energy-loss spectroscopy. Appl. Phys. Lett. 108, 093108 (2016).

33. Reed, W. A., Eisenber., P., Pandey, K. C. \& Snyder, L. C. Electron momentum distributions in graphite and diamond and carboncarbon bonding. Phys. Rev. B 10, 1507-1515 (1974).

34. Meyer, J. C. et al. Accurate measurement of electron beam induced displacement cross sections for single-layer graphene. Phys. Rev. Lett. 108, 196102 (2012). 


\section{Acknowledgements}

The authors acknowledge financial support from the National Natural Science Foundation of China (Grant No. 11504386), Key Research and Development Project of Shandong Province (Public Welfare Science and Technology Research, No. 2019GGX103010) and the Initial Foundation for Doctor Program of Liaocheng University (Grant No. 318051613). We thank Sara Maccagnano-Zacher, PhD, from Liwen Bianji, Edanz Editing China (www.liwenbianji.cn/ac), for editing the English text of a draft of this manuscript.

\section{Author contributions}

X.Z. and Z.F. performed EELS experiments. Y.S. did FLAPW calculations. H.L., Z.W. and H.H. analyzed the data and made figures. Z.F. and X.Z. wrote the paper. All authors had read and approved the final manuscript.

\section{Competing interests}

The authors declare no competing interests.

\section{Additional information}

Correspondence and requests for materials should be addressed to Z.F.

Reprints and permissions information is available at www.nature.com/reprints.

Publisher's note Springer Nature remains neutral with regard to jurisdictional claims in published maps and institutional affiliations.

(c) (i) Open Access This article is licensed under a Creative Commons Attribution 4.0 International License, which permits use, sharing, adaptation, distribution and reproduction in any medium or format, as long as you give appropriate credit to the original author(s) and the source, provide a link to the Creative Commons license, and indicate if changes were made. The images or other third party material in this article are included in the article's Creative Commons license, unless indicated otherwise in a credit line to the material. If material is not included in the article's Creative Commons license and your intended use is not permitted by statutory regulation or exceeds the permitted use, you will need to obtain permission directly from the copyright holder. To view a copy of this license, visit http://creativecommons.org/licenses/by/4.0/.

(C) The Author(s) 2019 